\title{
Nanoparticles Promise New Methods to Boost Oncology Outcomes in Breast Cancer
}

\author{
Jalil Pirayesh Islamian¹, Milad Hatamian ${ }^{1 *}$, Mohammad Reza Rashidi ${ }^{2}$
}

\begin{abstract}
Different types of treatment are available for patients with breast cancer, the most being radiotherapy, chemotherapy, hormonal therapy and combination therapy. Recently, nanoparticles have been emerging as promising agents for cancer therapy and are being investigated as contrast agents, drug carriers, radiosensitizers and also for hyperthermia effects. In this review the focus is on approaches for targeted treatment of breast cancer by combining nanoparticles, chemodrugs and radiation. The availble data suggest the possibility of increased roles for combined therapy, particularly by reducing the dose of each treatment modality, and consequently minimizing related side effects.
\end{abstract}

Keywords: Breast cancer - chemodrugs - doxorubicin - nanoparticles - radiation therapy - 2-deoxy-D-glucose

Asian Pac J Cancer Prev, 16 (5), 1683-1686

\section{Introduction}

Breast cancer is the most frequently diagnosed cancer in women and is the major cause of cancer death among women worldwide ( Elstner et al., 2002; Kaabinejadian et al., 2008; Loh et al., 2011). common treatment modality of breast cancer involve surgery, chemotherapy, radiation therapy, hormonal therapy, or combination therapy. Unfortunately, some limitation exists in this therapeutic methods such as radio-drug resistance, being special for certain type of cancer, undesirable injury to normal tissue surrounding of the tumor and etc; hold forth researchers attempt to improve methods of therapy to overcome at this limitation (such as use more sensitized compound, more localization of tumor, increase toxicity of chemodrug with reducing in side effect and etc.) and novel strategies require to boost the oncologic outcome (Aghaee et al., 2012; 2013; Jain et al., 2014).

Nowadays, nanoscience has emerged as one of the most hopeful application in medicine to enhance medical imaging output and also improve the rate of cancer therapy. In the field of cancer therapy most attention is investigated nanoparticles as a drug carrier, hyperthermia inducer, and also radiosensitizer agent (Selim and Hendi, 2012; Verma et al., 2013; El-Kassas and El-Sheekh, 2014; Jain et al., 2014; Nogueira et al., 2014).

\section{Breast Cancer}

The common risk factors for breast cancer includes: age (only, age at menarche and menopause, and age at first pregnancy), geographical variation, family history of breast cancer, hormonal mechanism, previous benign breast disease, radiation, life style, high fatty diet, oral contraceptive (McPherson et al., 2000; Aghaee et al., 2012).

In the field of medical imaging, common methods for diagnosis of breast cancer involved mammography, MRI, ultrasound and PET/CT (Yang et al., 2008).

In treatment of cancer after conservative surgery, radiation therapy is now of routine value to diminish regional tumor recurrence. In addition, closely $60 \%$ of all cancer treatments include radiation (alone or with surgery and chemotherapy). Furthermore, some limitation such as undesirable damage to normal, healthy tissue, presence of hypoxic and intrinsically radio resistance tumor cells limited the utilization of radiation therapy; moreover, chemotherapy immediately helps to radiotherapy by means of localization and sensitization of tumor with ionizing radiation (Cobb et al., 1996; Dwarkanath et al., 2001; Schwarz et al., 2008; Ghilotti et al., 2010; Aghaee et al., 2012).

In cancer chemotherapy, today's active researches are focused on taking advantage of biochemical differences between cancer cells and normal cell metabolism, such as energy metabolism to generated ATP for aerobic glycolysis from oxidative phosphorylation (Cheng et al., 2012). Enhance in the rate of glucose uptake and glycolysis as compared to corresponding normal tissue are characterization of malignant tumor(Warburg; Mentis et al., 2010). Studies suggest that in human cancer cell via metabolic oxidative stress, glucose deprivation can induce cytotoxicity (Ahmad et al., 2010).

2-Deoxy-D-Glucose (2DG) is a structural analog of

${ }^{1}$ Department of Medical Physics, Faculty of Medicine, ${ }^{2}$ Department of Medicinal Chemistry, Faculty of Pharmacy, Tabriz University of Medical Sciences, Tabriz, Iran*For correspondence: milad_hatamian@yahoo.com 
glucose and is one of functional chemodrug that is best known as an inhibitor of glucose metabolism. In vitro and in vivo studies were shown that $2 \mathrm{DG}$ is cytotoxic and also is known as a tumor sensitizer to irradiation and chemotherapy, which help improve the rates of therapy and capable induce radiosensitization in human cancer cells by means of glucose deprivation (Ahmad et al., 2010; Aghaee et al., 2012).

2DG undergoes expedite diffusion into cell via glucose pathway. Inside the cell, 2DG alternation to 2DG-6phosphate by hexokinase phosphorylation, and this formation of 2DG, 2DG-6-P, is not further metabolized by cell and the pentose phosphate pathway gets reduced, consequently 2DG-6-P accumulated in the cell until dephosphorylated by phosphorylase (Aghaee et al., 2012).

Doxorubicin (Dox) is one of the most toxic chemodrug by generating reactive oxygen species however the use of this drug limited for several side effects such cardiomyopathy (Takanashi and Bachur, 1976; Kong and Lillehei, 1998; Quiles et al., 2002). In a study of combining 2DG and Doxorubicin they conclude combination of 2DG Dox can be increase toxicity and reduce side effect of Doxorubicin with utilizing the low dose of Doxorubicin (Ahmad et al., 2010; Aghaee et al., 2013).

\section{Nanoparticles in Medical Imaging as Contrast Agents}

In the field of medical imaging, to enhance image contrast, nanoparticles were used as contrast agents. In a study on computed tomography (CT), 2DG labeled gold nanoparticles (AuNP) was studied as a contrast enhancement, and achieved significant contrast improvement in the A-549 liver cancer cell lines regarding to the unlabeled AuNP in multiple CT slice (Aydogan et al., 2010).

Other studies have also performed in the field of medical imaging such as magnetic nanoparticles for MRI improvement (Lu et al., 2009; Yen et al., 2013), silica nanoparticles for ultrasound imaging (Casciaro et al., 2010), improve sensitivity of PET signal (Nahrendorf et al., 2008) and gold nanoparticles for X-ray contrast enhancement (Hainfeld et al., 2014) and etc.

\section{Nanoparticles as drug carrier}

Recent studies were demonstrated that labeling nanoparticles with chemo drug not only improve the rate of therapeutic and enhance localization of the tumor cells but also reduce side effects of the chemodrugs (Mitra et al., 2001; Jain et al., 2014).

In the previous study experimental data shown that conjugating tio 2 with Doxorubicin increase the Doxorubicin localization in tumor cells, improve anticancer activity and also reduce toxic side effects of the Doxorubicin chemo drug (Chen et al., 2011).

In another study of Paclitaxel as one of the highly toxic anti cancer drug with adverse effects in healthy tissue, they were used $\mathrm{N}$-isopropylacrylamide/ vinyl pyrrolidone as polymeric nano drug carrier on the MCF7 breast carcinoma and $\mathrm{B} 16 \mathrm{~F} 0$ skin cancer cell line and show high therapeutic efficiency of nano loaded Paclitaxel by safe delivery to the cancer cells and sustained release action for the longest period of time and reduce the side effects (Yadav et al., 2014).

In vivo study in mouse models with curcumin loaded nanoparticles also resulted that curcumin-nanoparticles were effective to inhibit the growth of human lung cancer with little toxicity to normal tissue while the poor solubility of curcumin limits its further application in the treatment of cancer (Yin et al., 2013).

\section{Nanoparticles Induce Hyperthermia}

Hyperthermia widely used to induce apoptosis in many tissue and also to increase localized treatment in combination with chemo drug, radiation, radiofrequency waves, micro waves or ultrasound; but some limitation, such as lack of specifically for tumor tissue, has been restrict hyperthermia (Vernon et al., 1996; van der Zee et al., 2000; Wust et al., 2002; Issels et al., 2010).

In recent years, study on role of nanoparticles to resolve restriction of this problem have shown that magnetic nanoparticles such as iron oxide nanoparticles could be a useful technique for local hyperthermia treatment of cancer (Basel et al., 2012). In the same study, researchers applied magnetic Hydroxyapatite nanoparticles on a mouse model and demonstrated therapeutic effects in vivo with little toxicity at normal tissue (Hou et al., 2009).

\section{Nanoparticles as Radiosensitizers}

Less toxicity and also less injury to healthy surrounding tissues of the tumor and delivery high accuracy of damage with ionization radiation or visible light in cancer treatment, lead researchers to focus on developing photosensitizer such as quantum dots for superficial tumor with visible light and also nanoparticles as radiosensitizer with ionization radiation for patients with tumor in internal organs (Juzenas et al., 2008).

In a study on human prostate carcinoma cell line, bonding glucose with gold nanoparticles it was demonstrated that gold nanoparticles with or without bonding to glucose enhances radiosensitivity on prostate cancer cell line. Furthermore, there were significantly increase in cell uptake and also enhanced cell killing effect compared to gold nanoparticles without glucose bonding (Zhang et al., 2008).

In a research on linking gold nanoparticles with DNA scaffold it has been shown enhancement of radiosensitivity caused by an increased absorption of radiation energy by means of gold nanoparticles, also very few gold nanoparticles bounded to DNA molecule are needed to considerably increase the damage to this molecule by a $60 \mathrm{keV}$ electron beam; however the role of high energy particles cannot be ignored in the study (Zheng et al., 2009).

In vitro study on the size of gold nanoparticles demonstrated that $1.9 \mathrm{~nm}$ gold nanoparticles were uptaked by breast cancer cell lines and caused radiosensitivity with $\mathrm{kV}$ and $\mathrm{MV}$ photons in cell lines, but this radiosensitization is cell dependent. Also this effect was observed in MDA- 
MB-231 cells, but not observed in DU-145 or L-132 breast cancer cell lines in spite of uptake take place in this cell lines. In addition, sensitization of MDA-MB-231 cells was shown at $\mathrm{kV}$ and $\mathrm{MV}$ photon energy and also with MV electrons which is inconsistent with prognostication from Monte Carlo simulation modeling (Cho, 2005; Jain et al., 2011).

\section{Conclusion}

The application of nanoparticles may an effective approach in induction of apoptosis and also preventing of tumor cell growth. On the other hand, the coating materials used for nanoparticle have demonstrated to enhance radiosensitivity on the tumor cells. The other mechanisms related for nanoparticles are producing local hyperthermia which resulting in tumor cell necrosis. Doxorubicin is considered to be the most effective agent in the treatment of breast cancer patients. Unfortunately, resistance to this agent and also severs cardiotoxicity are common, representing a major obstacle to successful treatment. 2-Deoxy-D-Glucose causes cytotoxicity in cancer cells by disrupting thiol metabolism while for Doxorubicin by generating reactive oxygen species. Research activity aimed towards developing and improving nanoparticle targeting systems has expanded tremendously in the past years with new ways of delivering the drugs to tumors as well as new types of drugs. Therefore, combined nanoparticle with anticancer drugs, 2-Deoxy-D-Glucose and Doxorubicin, can have the benefit of increased breast cancer cure by means of more localization chemodrug, enhanced radiosensitization, increased cytotoxicity in tumor cells, decreased the dose used for radiation and chemodrug and also decreased side effects of chemoradiotherapy.

\section{Acknowledgements}

This review is a part of literature review for a research grant by the Research Center for Pharmaceutical Nanotechnology in Tabriz University of Medical Sciences (\# 5.87.596), Tabriz, Iran.

\section{References}

Aghaee F, Islamian JP, Baradaran B, et al (2013). Enhancing the effects of low dose doxorubicin treatment by the radiation in T47D and SKBR3 breast cancer cells. J Breast Cancer, 16, 164-70.

Aghaee F, Pirayesh Islamian J, Baradaran B (2012). Enhanced radiosensitivity and chemosensitivity of breast cancer cells by 2-deoxy-d-glucose in combination therapy. $J$ Breast Cancer, 15, 141-7.

Ahmad IM, Mustafa EH, Mustafa NH, et al (2010). 2DG enhances the susceptibility of breast cancer cells to doxorubicin. Cent Eur J Biol, 5, 739-48.

Aydogan B,Li J, Rajh T, et al (2010). AuNP-DG: deoxyglucoselabeled gold nanoparticles as X-ray computed tomography contrast agents for cancer imaging. Mol Imaging Biol, 12, 463-7.

Basel MT, Balivada S, Wang H, et al (2012). Cell-delivered magnetic nanoparticles caused hyperthermia-mediated increased survival in a murine pancreatic cancer model. Int J Nanomedicine, 7, 297.

Casciaro S, Conversano F, Ragusa A, et al (2010). Optimal enhancement configuration of silica nanoparticles for ultrasound imaging and automatic detection at conventional diagnostic frequencies. Invest Radiol, 45, 715-24.

Chen Y, Wan Y, Wang Y, et al (2011). Anticancer efficacy enhancement and attenuation of side effects of doxorubicin with titanium dioxide nanoparticles. Int J Nanomedicine, 6, 2321.

Cheng G, Zielonka J, Dranka BP, et al (2012). Mitochondriatargeted drugs synergize with 2-deoxyglucose to trigger breast cancer cell death. Cancer Res, 72, 2634-44.

Cho SH (2005). Estimation of tumour dose enhancement due to gold nanoparticles during typical radiation treatments: a preliminary Monte Carlo study. Phys Med Biol, 50, 163.

Cobb JP, Hotchkiss RS, Karl IE, et al (1996). Mechanisms of cell injury and death. Br J Anaesth, 77, 3-10.

Dwarkanath BS,ZolzerF, Chandana S, et al (2001). Heterogeneity in 2-deoxy-D-glucose-induced modifications in energetics and radiation responses of human tumor cell lines. Int $J$ Radiat Oncol Biol Phys, 50, 1051-61.

El-Kassas HY, El-Sheekh MM (2014). Cytotoxic activity of biosynthesized gold nanoparticles with an extract of the red seaweed Corallina officinalis on the MCF-7 human breast cancer cell line. Asian Pac J Cancer Prev, 15, 4311.

Elstner E, Williamson EA, Zang C, et al (2002). Novel therapeutic approach: ligands for PPAR $\gamma$ and retinoid receptors induce apoptosis in bcl-2-positive human breast cancer cells. Breast Cancer Res Treat, 74, 155-65.

Ghilotti M, Pierotti MA, Gariboldi M (2010). Molecular markers for prediction of risk of radiation-related injury to normal tissue. J Nucleic Acids Investig, 1, 11.

Hainfeld JF, Slatkin DN, Focella TM, et al (2014). Gold nanoparticles: a new X-ray contrast agent. Br J Radiol, 79, 248-53.

Hou CH, Hou SM, Hsueh YS, et al (2009). The in vivo performance of biomagnetic hydroxyapatite nanoparticles in cancer hyperthermia therapy. Biomaterials, 30, 3956-60.

Issels RD, Lindner LH, Verweij J, et al (2010). Neo-adjuvant chemotherapy alone or with regional hyperthermia for localised high-risk soft-tissue sarcoma: a randomised phase 3 multicentre study. Lancet Oncol, 11, 561-70.

Jain S, Coulter JA, Hounsell AR, et al (2011). Cell-specific radiosensitization by gold nanoparticles at megavoltage radiation energies. Int J Radiat Oncol Biol Phys, 79, 531-9.

Jain S, Hirst DG, O'Sullivan JM (2014). Gold nanoparticles as novel agents for cancer therapy. Br J Radiol, 85, 101-13.

Juzenas P, Chen W, Sun Y-P, et al (2008). Quantum dots and nanoparticles for photodynamic and radiation therapies of cancer. Adv Drug Deliv Rev, 60, 1600-14.

Kaabinejadian S, Fouladdel SH, Ramezani M, et al (2008). p53 expression in MCF7, T47D and MDA-MB 468 breast cancer cell lines treated with adriamycin using RT-PCR and immunocytochemistry. J Biol Sci, 8, 380-5.

Kong Q, Lillehei KO (1998). Antioxidant inhibitors for cancer therapy. Med Hypotheses, 51, 405-9.

Loh SY, Chew SL (2011). Awareness and practice of breast self examination among Malaysian women with breast cancer. Asian Pac J Cancer Prev, 12,199-202.

Lu J, Ma S, Sun J, et al (2009). Manganese ferrite nanoparticle micellar nanocomposites as MRI contrast agent for liver imaging. Biomaterials, 30, 2919-28.

McPherson K, Steel C, Dixon JM (2000). ABC of breast diseases: breast cancer-epidemiology, risk factors, and genetics. $B M J$, 321, 624.

Mentis A-FA, Saha P, Das S, et al (2010). Metabolism and cancer: 
an up-to-date review of a mutual connection. Asian Pac J Cancer Prev, 11, 1437-44.

Mitra S, Gaur U, Ghosh PC, et al (2001). Tumour targeted delivery of encapsulated dextran-doxorubicin conjugate using chitosan nanoparticles as carrier. J Control Release, 74, 317-23.

Nahrendorf M, Zhang H, Hembrador S, et al (2008). Nanoparticle PET-CT imaging of macrophages in inflammatory atherosclerosis. Circulation, 117, 379-87.

Nogueira DR, Rolim CMB, Farooqi AA (2014). Nanoparticle induced oxidative stress in cancer cells: adding new pieces to an incomplete jigsaw puzzle. Asian Pac J Cancer Prev, 15, 4739-43.

Quiles JL, Huertas JR, Battino M, et al (2002). Antioxidant nutrients and adriamycin toxicity. Toxicol, 180, 79-95.

Schwarz SB, Schaffer PM, Kulka U, et al (2008). The effect of radio-adaptive doses on HT29 and GM637 cells. Radiat Oncol, 3, 1-6.

Selim ME, Hendi AA (2012). Gold nanoparticles induce apoptosis in MCF-7 human breast cancer cells. Asian Pac J Cancer Prev, 13, 1617-20.

Takanashi S, Bachur NR (1976). Adriamycin metabolism in man. Evidence from urinary metabolites. Drug Metab Dispos, 4, 79-87.

Van der Zee J, González D, van Rhoon GC, et al (2000). Comparison of radiotherapy alone with radiotherapy plus hyperthermia in locally advanced pelvic tumours: a prospective, randomised, multicentre trial. Lancet, $\mathbf{3 5 5}$, 1119-25.

Verma NK, Crosbie-Staunton K, Satti A, et al (2013). Magnetic core-shell nanoparticles for drug delivery by nebulization. J Nanobiotechnol, 11.

Vernon CC, Hand JW, Field SB, et al (1996). Radiotherapy with or without hyperthermia in the treatment of superficial localized breast cancer: Results from five randomized controlled trials. Int J Radiat Oncol Biol Phys, 35, 731-44.

Warburg O (1956). The metabolism of tumors, Science, 123, 309-14.

Wust P, Hildebrandt B, Sreenivasa G, et al (2002). Hyperthermia in combined treatment of cancer. Lancet Oncol, 3, 487-97.

Yadav D, Anwar MF, Garg V, et al (2014). Development of polymeric nanopaclitaxel and comparison with free paclitaxel for effects on cell proliferation of MCF-7 and B16F0 carcinoma cells. Asian Pac J Cancer Prev, 15, 2335-40.

Yang WT, Le-Petross HT, Macapinlac H, et al (2008). Inflammatory breast cancer: PET/CT, MRI, mammography, and sonography findings. Breast Cancer Res Treat, 109, 417-26.

Yen SK, Padmanabhan P, Selvan ST (2013). Multifunctional iron oxide nanoparticles for diagnostics, therapy and macromolecule delivery. Theranostics, 3, 986.

Yin H-T, Zhang DG, Wu XL, et al (2013). In vivo evaluation of curcumin-loaded nanoparticles in a A549 xenograft mice model. Asian Pac J Cancer Prev, 14, 409-12.

Zhang X, Xing JZ, Chen J, et al (2008). Enhanced radiation sensitivity in prostate cancer by gold-nanoparticles. Clin Invest Med, 31, 160-7.

Zheng Y, Hunting DJ, Ayotte P, et al (2009). Radiosensitization of DNA by gold nanoparticles irradiated with high-energy electrons. Radiat Res, 169, 19-27. 